\title{
ADAPTIVE STRATEGY-BASED TIGHTLY-COUPLED INS/GNSS INTEGRATION SYSTEM AIDED BY ODOMETER AND BAROMETER
}

\author{
Y. C. Tien ${ }^{1 *}$, Y. L. Chen ${ }^{1}$, K. W. Chiang ${ }^{1}$ \\ ${ }^{1}$ Dept. of Geomatics, National Cheng-Kung University, No. 1, Daxue Road, East District, Tainan 701, Taiwan - \\ P66064162@mail.ncku.edu.tw; eltonboy78@gmail.com; kwchiang@mail.ncku.edu.tw
}

KEY WORDS: INS/GNSS, Tightly-coupled scheme, Odometer, Barometer, Adaptive Kalman Filter

\begin{abstract}
:
Inertial Navigation System/Global Navigation Satellite System (INS/GNSS) integration system have been widely applied in recent years. Unfortunately, it sometimes malfunctions and the performance heavily deteriorates, especially in urban area where signals from satellites may be blocked or reflected by modern buildings. In multipath or Non Light-of-sight (NLOS) environment, incorrect signal results in poor observability of GNSS measurement model in Kalman Filter (KF). For purpose of addressing the issue, we proposed an adaptive strategy-based tightly-coupled INS/GNSS integration system aided by odometer and barometer, targeting to mitigate the error from poor observability. In this method, tightly-coupled (TC) scheme is implemented as the fundamental system in order to increase the reliability and stability. TC is more suitable than Loosely-coupled (LC), the traditional scheme, in urban navigation because it requires less visible GNSS measurement and it overcomes the disadvantage of LC, and further enhances the navigation result. Furthermore, aiding sensors such as odometer and barometer are integrated in this system as well, serving as velocity and height constraints respectively. Since the precision of GNSS positioning depends on the properties of the environment, measurement model of KF must work adaptively. Thus, innovation-based Adaptive Scaled Estimation (IASE) and Residual-based Adaptive Scaled Estimation (RASE), are also implemented to improve navigation performance in this paper. Finally, from the experimental validation, the proposed adaptive sensor-fusion navigation algorithm significantly enhanced the performance. The improvement was approximate $80 \%$ compared with the pure TC scheme; the RMSE can reach $6 \mathrm{~m}$ in $3 \mathrm{D}$ and $2.5 \mathrm{~m}$ in vertical.
\end{abstract}

\section{INTRODUCTION}

Due to the ever-increasing demand and application of geospatial information and Location-Based Service (LBS), positioning technology has become more and more popular in human daily lives and lots of researches were done to increase the accuracy and efficiency of navigation technique. The determination of position, velocity and attitude of an object is the definition of navigation (Farrell et al., 1998). With the position and other geospatial information of vehicles, application such as Intelligent Transport System (ITS) and smart city can be fulfil and achieved, which will increase the convenience and efficiency of human lives. Intelligent vehicle will be the most important key element for the future smart city and it requires high performance navigation technologies (Sun et al., 2016). To realize an intelligent vehicle, Global Navigation Satellite System (GNSS) and its integration with multi-sensors have been widely applied. Currently, the famous and commonly used navigation technique is Inertial Navigation System/Global Navigation Satellite System (INS/GNSS) integration system, which provides the precise and continuous positioning result with high sampling frequency, even when in GNSS signal outages. The combination of INS and GNSS is able to overcome the drawbacks of individual system. Therefore, INS/GNSS integration not only promotes the quality and performance of navigation but also increases the reliability (Roger, 2003).

However, the performance of INS/GNSS integration depends heavily on the quality of Inertial Measurement Unit (IMU) adopted. The positioning accuracy will degrade rapidly while GNSS interruption and blockage if only low cost IMU is applied (M. Park and Y. Gao, 2008). Although adoption of high-end tactical IMU makes system more stable and reliable, the price and cost is the major difficulty to apply tactical IMU diffusely. As a result, one of the efficient ways to enhance the accuracy is to refine the integration scheme as well as to aid the navigator with complementary sensor such as odometer and barometer.
For the core of INS/GNSS integration structure, Loosely-coupled (LC) integration scheme has been the most popular strategy due to its simplicity structure, easy feasibility and less computation burden. LC requires the position and velocity information from GNSS (i.e. navigation solution) to calibrate the error of INS.

Since GNSS provides worldwide and drift-free absolute positioning result and service (Yang, 2008), many papers have present interests on the $\mathrm{LC}$ and its real-time applications (Petovello, 2003, Shin, 2005 and so on). The structure of LC scheme is shown Figure 1. Note that Figure 1 is simplified for the viewpoints of this paper, refer to (Chiang et al, 2013) for details. However, the fatal weakness of LC is that outage of GNSS solution makes it an INS stand-alone system especially in modern urban canyons. This troubles LC when in the harsh urban environments and make it unsuitable for future smart city and ITS application.

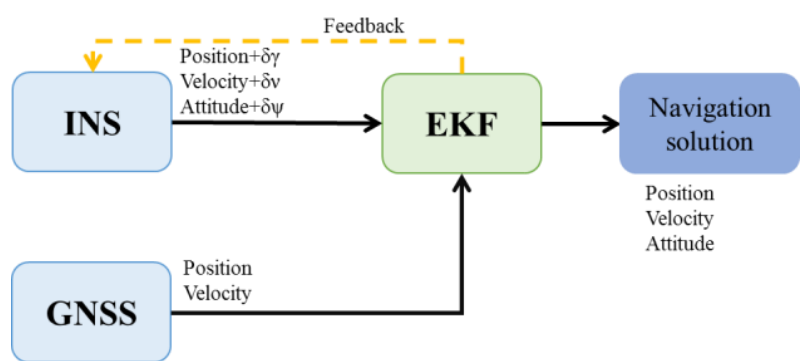

Figure 1. Structure of LC scheme

GNSS signal blockage takes place frequently in modern urban area due to severe signal obstruction in urban canyons. In addition, the height component of GNSS solution suffers from significant oscillation mainly resulted from the impact of multipath (Petovello, 2003), Non Light-of-Sight (NLOS) (Hsu et al., 2015) and poor geometry in vertical. The reflected or blocked signals will confuse the system by incorrect measurement and 
then lead to degrade of positioning estimation and even no positioning solution. The multipath and NLOS effect is demonstrated in Figure 2.

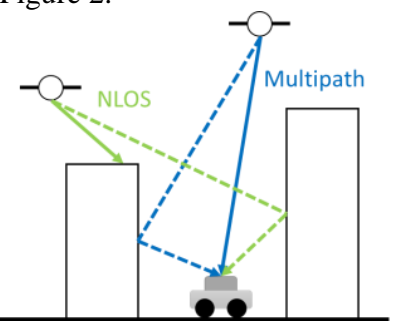

Figure $\overline{2 \text {. Multipath (blue) and NLOS (green) }}$

In order to reduce the frequency of absent GNSS solution and distorted one, another integration scheme called Tightly-coupled (TC) scheme is proposed and it is adopted as the fundamental core in this paper. TC scheme exploits the raw measurement of GNSS, pseudorange and pseudorange rate, directly, which means that TC keeps on-line calibrating INS error while any raw measurement is available. Even though the vehicles are in urban canyons, TC keeps update loops by receiving measurement of visible satellites with high elevation angle. The structure of TC scheme is shown Figure 3. Note that Figure 3 is simplified for the viewpoints of this paper, refer to (Chiang et al, 2013) for details.

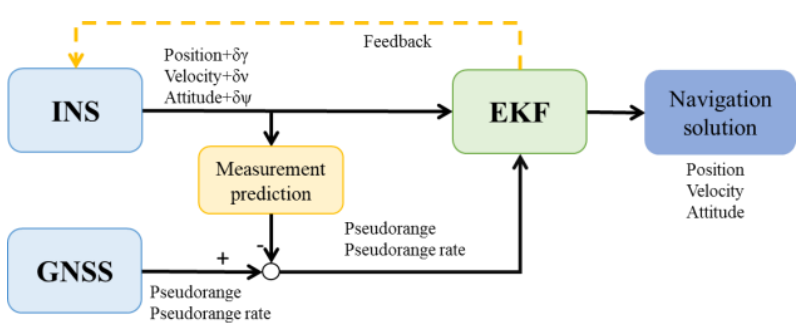

Figure 3. Structure of TC scheme

Although TC scheme reduces the absence of GNSS update in Kalman Filter (KF), there is still another drawback of TC in harsh urban area (Chiang and Huang, 2008). Since TC directly exploits the pseudorange and pseudorange rate in measurement model, the accuracy of estimation is subject to the quality of raw measurement of GNSS, which will be affected by multipath and NLOS effect especially in urban canyons. To accurately adjust for the statistical characteristics of the system model, measurement model and noise, adaptive strategies for $\mathrm{KF}$ measurement model should be adopted for autonomous tuning (Almagbile et al., 2010; Pemg et al., 2013). KF with an adaptive strategy which is also known as Adaptive Kalman Filter (AKF) incorporates the uncertainty of covariance matrix of measurement error ( $\mathrm{R}$ matrix) and covariance matrix of system noise ( $Q$ matrix) for adaptive model tuning, which can adjust the weight of each measurement. AKF is able to reduce the weight of bad measurements with poor geometry in KF update loops and therefore suppresses the impact of them on filtered solutions. In order to achieve reasonable balance of measurement model and system model, several adaptive strategies have been applied. Wu and Yang (2013) used amount of visible satellite and PDOP as index to enable adaptive method for TC scheme; Liu at al (2017) implemented AKF based on innovation sequence and the attenuation factor in a moving window; Hajiyev et al (2016) adopted innovation-based Adaptive Scaled Estimation (IBASE) and Residual-based Adaptive Scaled Estimation (RBASE) methods. Considering the robustness and statistical characteristics, IBASE and RBASE are implemented in this paper due to the more stable and healthier procedure.
As for land vehicle navigations, odometer and barometer were widely used as aiding sensors (Sokolovic at al., 2013 Park et al., 2015). An odometer is a velocity sensor and it is self-contained and it provides undisrupted velocity information continuously. It, in general, is more accurate and frequent velocity measurement than the GNSS. Odometer can serve as a continuous measurement to calibrate IMU error, no matter the change of outside environment. A barometer is a self-contained altitude sensor and it provides height information. As an aiding, barometer produces continuous height information with higher frequency than GNSS does as height constraints, mitigating the weakness of GNSS positioning in vertical direction.

As a result, an adaptive strategy-based tightly-coupled INS/GNSS integration system aided by odometer and barometer for integrating low-cost MEMS sensors and GNSS for seamless land vehicular application is proposed in this study. For the system performance assessment and evaluation, field tests are conducted with many urban scenarios. The main objectives of this paper are to: (1) develop an INS/GNSS TC integration scheme for seamless intelligent land vehicular navigation; (2) implement and verify the odometer and barometer aiding schemes; (3) employ and implement adaptive strategies for INS/GNSS TC integration system and (4) analysis the effectiveness and performance of proposed TC AKF with aiding sensor.

\section{METHODOLOGY}

A proposed adaptive strategy-based tightly-coupled INS/GNSS integration system aided by odometer and barometer, targeting to mitigate the error resulting from poor GNSS signal observability in urban area is implemented in this paper. Figure 4 describes the structure of proposed navigation algorithm. First INS generates position, velocity and attitude with unbounded error through the INS mechanization (Titterton at al., 1994), and its estimation result can be used to do measurement prediction through $\mathrm{KF}$ measurement model. For GNSS part, it provides pseudorange and pseudorange rate, which is the actual measurement. Next, both predicted and practical measurements are then compared to calculate the innovation sequence, which is the amount of new information being introduced into KF from practical measurement (Petovello, 2003). With this innovation sequence, specific adaptive strategy for KF system noise model and measurement error model re-weights the measurement accordingly. In other words, AKF modifies the covariance matrix of system noise ( $Q$ matrix) and the covariance matrix of measurement error ( $\mathrm{R}$ matrix) in accordance with outer environment. Furthermore, additional sensors including barometer and odometer provide height information and velocity measurement respectively, serving as aiding constraints for optimization of INS/GNSS integration system. Aiding sensors always bring more information into $\mathrm{KF}$, which tremendously reduces the duration that INS is left stand-alone, even if under hard conditions such as no GNSS measurement available. The aiding sensors not only provide additional measurement when GNSS signal is available, but also keep calibrating and restricting the large growth of INS error when lack of GNSS signal such indoor parking lots.

In this paper, we focus on the core of navigator, i.e. the AKF block in Figure 4. In AKF, all information are blended to optimize the solution through a series of prediction and update loops. Eventually, improved and enhanced navigation solution are generated. Following sections depict two main procedures of the proposed navigation algorithm, including tightly coupled integration scheme and adaptive strategy. Note that some parts of Figure 4 are simplified for the viewpoints of this paper. 


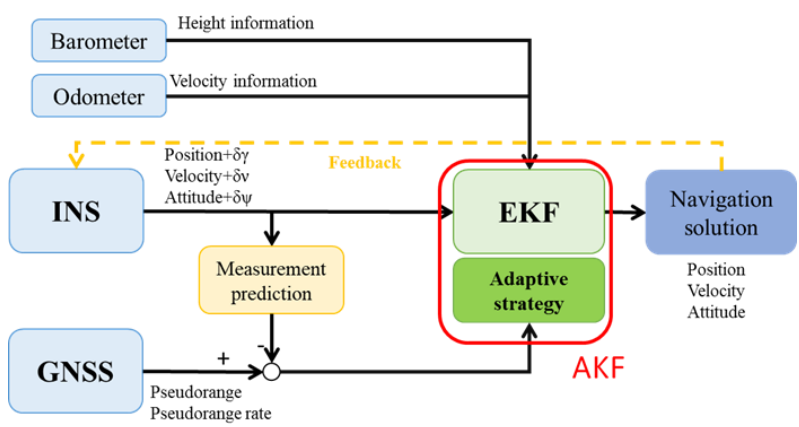

Figure 4. Structure of TC aided AKF

\subsection{Tightly-Coupled Integration Scheme}

\subsubsection{Kalman filtering}

The time-discrete dynamic equation which describes how the state vector evolves (Gelb, 1974) with time is given as Eq.1. On the other hand, the measurement model is given as Eq.2. In the measurement model, predicted measurement is generated through the design matrix and state vector.

$$
\begin{gathered}
\mathrm{x}_{\mathrm{k}+1}=\Phi_{k, k+1} x_{k}+G_{k} \omega_{k} \\
\mathrm{z}_{\mathrm{k}}=\mathrm{H}_{\mathrm{k}} \mathrm{x}_{\mathrm{k}}+v_{\mathrm{k}}
\end{gathered}
$$

where subscript " $k$ " indicates the timestamp

$\mathrm{x}=$ the state vector

$\Phi_{\mathrm{k}, \mathrm{k}+1}=$ transition matrix from epoch $\mathrm{k}$ to $\mathrm{k}+1$

$\mathrm{G}_{\mathrm{k}}=$ shaping matrix at epoch $\mathrm{k}$

$\omega_{\mathrm{k}}=$ system noise at epoch $\mathrm{k}$

$\mathrm{Z}_{\mathrm{k}}=$ measurement at epoch $\mathrm{k}$

$\mathrm{H}_{\mathrm{k}}=$ the design matrix at epoch $\mathrm{k}$

$v_{\mathrm{k}}=$ the measurement error

As mentioned before, $\mathrm{KF}$ executes a series of prediction and update stages. In the prediction stage, the state vector and the associated covariance matrix of system noise are predicted, based on state vector from last epoch and the transition matrix. The prediction procedure contains following equations:

$$
\begin{gathered}
\hat{\mathrm{x}}^{-}{ }_{k+1}=\Phi_{k, k+1} \hat{x}^{+}{ }_{k} \\
\mathrm{P}_{\mathrm{k}+1}^{-}=\Phi_{k, k+1} P_{k}^{+} \Phi_{k, k+1}^{T}+Q_{k}
\end{gathered}
$$

where variables with a hat “^” is estimated value superscript "-" is Kalman predicted quantity superscrip "+" is Kalman update quantity $\mathrm{P}_{\mathrm{k}}=$ covariance matrix of state vector at epoch $\mathrm{k}$ $\mathrm{Q}_{\mathrm{k}}=$ covariance matrix of system noise at epoch $\mathrm{k}$

The other stage depicting the measurement update of system model is update stage and the procedure is given as follows.

$$
\begin{gathered}
\mathrm{K}_{\mathrm{k}}=P_{k}^{-} H_{k}^{T}\left(H_{k} P_{k}^{-} H_{k}^{T}+R_{k}\right)^{-1} \\
\hat{\mathrm{x}}_{k}^{+}=\hat{x}_{k}^{-}+K_{k}\left(z_{k}-H_{k} \hat{x}_{k}^{-}\right) \\
\mathrm{P}_{\mathrm{k}}=\left(I-K_{k} H_{k}\right) P_{k}^{-}
\end{gathered}
$$

where $\mathrm{R}_{\mathrm{k}}=$ covariance matrix of measurement error at $\mathrm{k}$ $\mathrm{K}_{\mathrm{k}}=$ Kalman gain matrix $\mathrm{I}=$ identity matrix.
The overall work flow (Godha, 2006) is shown in Figure 5.

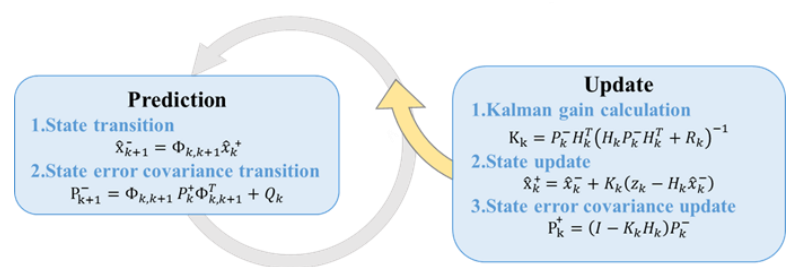

Figure 5. Flow chart of time-discrete KF stages

\subsubsection{System model and measurement Model}

In this paper, two kinds of single frequency GPS (Global Positioning System) measurements, code phase measurement and Doppler measurement, were used as observation. Code phase measurement is also known as pseudorange, and it is the observed distance between satellites and antenna. It is derived by calculating the time delay between GPS signal transmission and the reception, multiplied by the speed of signal (equal to the speed of light). Unfortunately, the theoretical range is heavily subject to the external environment and the hardware component, for example, atmospheric fraction and the imperfect time synchronization ability of the receiver. These error terms deteriorate the signal quality, and then put impact on the performance and the ranging accuracy. Error like receiver clock bias, distortion caused by ionosphere and troposphere delay must be considered and compensated correctly. Thus, the general code phase measurement equation comes as follows,

$$
\mathrm{P}=\mathrm{r}+C(d t+d T)+d_{\text {iono }}+d_{\text {trop }}+\epsilon
$$

where

$$
\begin{aligned}
& \mathrm{P}=\text { pseudorange measurement } \\
& \mathrm{r}=\text { theoretical distance between satellite and receiver } \\
& \mathrm{C}=\text { speed of light } \\
& \mathrm{dt} \text { is satellite clock bias } \\
& \mathrm{dT}=\text { receiver clock bias } \\
& \mathrm{d}_{\text {iono }}=\text { ionospheric delay } \\
& d_{\text {trop }}=\text { tropospheric delay } \\
& \epsilon=\text { other error term }
\end{aligned}
$$

On the other hand, the Doppler measurement represents the shift rate of carrier phase. This shift occurs because of relative movement between satellites and antenna (Chen, 1994). The relative velocity can be derived, as the Doppler Effect says, with prior knowledge of signal frequency. In the statement of GNSS, Doppler Effect is applied in order to estimate the rover velocity with given satellite velocity (Misra et al., 2001). The Doppler shift is derived based on the transmitted and received frequency and the equation comes as Eq.9.

$$
\begin{gathered}
\mathrm{D}_{\mathrm{i}}=f_{i}^{\text {receive }}-f_{i}^{\text {transmit }} \\
=-\frac{f_{i}^{\text {transmit }}}{C}\left[e_{i} \cdot\left(v_{s v_{i}}-v_{\text {rover }}\right)\right]
\end{gathered}
$$

where $\quad \mathrm{f}_{i}^{\text {receive }}=$ received signal frequency

$\mathrm{f}_{\mathrm{i}}^{\text {transmit }}=$ transmit signal frequency

$\mathrm{v}_{\mathrm{Sv}_{\mathrm{i}}}=$ velocity of the $\mathrm{i}^{\text {th }}$ observed satellite

$\mathrm{v}_{\text {rover }}=$ velocity of the rover

$\mathrm{e}_{\mathrm{i}}=$ line-of-sight vector

Note that the operation in the square bracket between $e_{i}$ and $\left(\mathrm{v}_{\mathrm{sv}_{\mathrm{i}}}-v_{\text {rover }}\right)$ is the dot product. Petovello et al (2003) said that the Doppler shift represents the frequency difference according to the relative motion and it is exactly a scale away from the 
pseudorange rate measurement. Hence, the measurement of pseudorange rate is written as Eq.10.

$$
\dot{\rho}_{i}=\left[\mathrm{e}_{\mathrm{i}} \cdot\left(\mathrm{v}_{\mathrm{sv}_{\mathrm{i}}}-\mathrm{v}_{\text {receiver }}\right)\right]+C(\dot{d} t-\dot{d} T)+\epsilon
$$

where $\quad \dot{\rho}_{i}=$ the $\mathrm{i}^{\text {th }}$ pseudorange rate measurement $\dot{d} t=$ the satellite clock drift

$\dot{d} T=$ the receiver clock drift.

In order to derive the measurement model of tightly-coupled scheme, the theoretical values of pseudorange and pseudorange rate should calculated first, thus, the general code phase and Doppler measurement model comes as follows,

$$
\begin{gathered}
\rho_{\mathrm{INS}, \mathrm{i}}=\left|\gamma_{I N S}^{e}-\left(\gamma_{S V}^{e}\right)_{i}\right| \\
\dot{\rho}_{I N S, i}=\frac{C_{e}^{n}\left\{\left(\gamma_{I N S}^{e}-\gamma_{S V, i}^{e}\right) \cdot\left(v_{I N S}^{e}-v_{S V, i}^{e}\right)\right\}}{\left(\rho_{I N S}\right)_{i}} \\
\mathrm{Z}_{\mathrm{TC}}=\left[\begin{array}{c}
\rho_{I N S, i}-\rho_{i} \\
\dot{\rho}_{I N S, i}-\dot{\rho}_{i}
\end{array}\right]_{2 n-3}
\end{gathered}
$$

where $\quad \rho_{I N S, i}=$ distance between receiver and the $\mathrm{i}^{\text {th }}$ satellite $\gamma_{\mathrm{INS}}^{e}=$ position vector of receiver in e-frame $\gamma_{S V, i}^{e,}=$ the position vector of $\mathrm{i}^{\text {th }}$ satellite in e-frame $\mathrm{C}_{\mathrm{e}}^{\mathrm{n}}=$ the rotation matrix from e-frame to $\mathrm{n}$-frame $v_{\text {INS }}^{e}=$ velocity vector of receiver in e-frame $v_{S V, i}^{e}=$ the velocity vector of $\mathrm{i}^{\text {th }}$ satellite in e-frame.

Besides, TC can estimate the receiver clock bias and drift. The state vector and the system model come as follows (Chiang at al., 2013).

$$
\begin{aligned}
& \delta \mathrm{x}_{11 \times 1}
\end{aligned}
$$

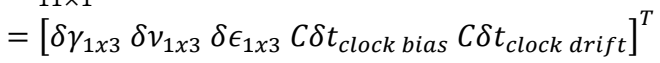

$$
\begin{aligned}
& \mathrm{C} \cdot\left[\begin{array}{c}
\delta \dot{t}_{\text {clock bias }} \\
\delta \dot{t}_{\text {clock drift }}
\end{array}\right]=\left[\begin{array}{ll}
1 & 0 \\
0 & 1
\end{array}\right]\left[\begin{array}{c}
C \delta t_{\text {clock bias }} \\
C \delta t_{\text {clock drift }}
\end{array}\right]+\mathrm{v}
\end{aligned}
$$

The design matrix of TC is shown in Eq.16.

$$
\mathrm{H}_{\mathrm{TC}}=\left[\begin{array}{ccccc}
\left(e_{i}\right)_{n \times 3} & 0_{n \times 3} & 0_{n \times 3} & 1 & 0 \\
0_{n \times 3} & 1 / \lambda\left(e_{i}\right)_{n \times 3} & 0_{n \times 3} & 0 & 1
\end{array}\right]_{2 n \times 11}
$$

where $\lambda=$ the signal wavelength depending on application $\mathrm{e}=$ the line-of-sight vector

$$
\mathrm{e}_{\mathrm{i}}=\frac{\left[\left(X_{I N S}-X_{S V_{i}}\right)\left(Y_{I N S}-Y_{S V_{i}}\right)\left(Z_{I N S}-Z_{S V_{i}}\right)\right]}{\left(\rho_{I N S}\right)_{i}}
$$

Where $\quad X_{\text {INS }}, Y_{\text {INS }}, Z_{\text {INS }}=$ position of IMU in e-frame $\mathrm{X}_{\mathrm{SVi}}, \mathrm{Y}_{\mathrm{SVi}}, \mathrm{Z}_{\mathrm{SVi}}=$ position of $\mathrm{i}^{\text {th }}$ satellite in e-frame

\subsection{Adaptive strategy}

Covariance matching, one of the parametric methods for adaptive problem, makes all elements of innovation-based or residual-based covariance matrix consistent with their theoretical values (Dimirovski, 2016; Almagbileet al., 2010). The unknown noise covariance of Kalman Filter can be determined by the statistical analysis of innovation or residual series, under assumption that the estimated covariance matrix of innovations or residuals should match their expected value. The flowchart of adaption strategy is demonstrated in Figure 6.

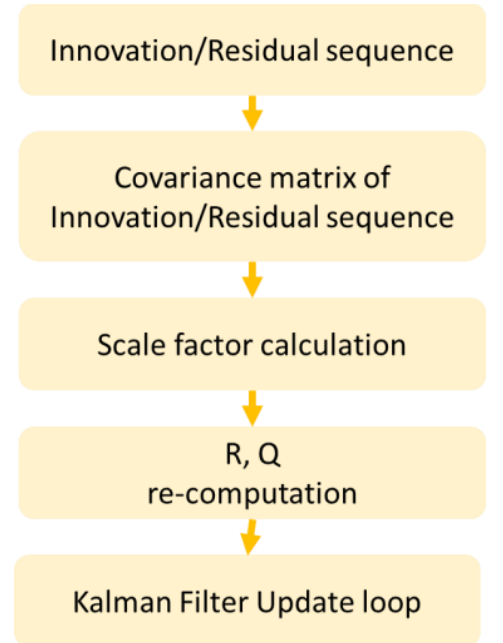

Figure 6. Flowchart of IBASE and RBASE

Innovation, $\mathrm{v}^{-}$and residual, $\mathrm{v}^{+}$mentioned here are computed by Eq.18 and Eq.19, respectively.

$$
\begin{aligned}
& v^{-}=z-H \hat{x}^{-} \\
& v^{+}=z-H \hat{x}^{+}
\end{aligned}
$$

Innovation vector serves as the foundation of Innovation-based adaptive scaling estimation (IASE), one of schemes of AKF. IASE method modifies Q, covariance matrix of system noise, and $\mathrm{R}$, covariance matrix of measurement error of Kalman Filter, based on the innovation covariance matrix, namely the outer environmental condition. Adaptive mechanism is enabled while the real value exceeds the expected one due to the significant change in operation condition of measurement (Dimirovski, 2016). The covariance matrix of innovation, $\widehat{C}_{v^{-}}$is calculated through averaging inside the moving window as Eq. 20.

$$
\hat{C}_{v^{-}}=\frac{1}{N} \sum_{j=j_{0}}^{k} \mathrm{v}_{\mathrm{j}}^{-} v_{j}^{-T}
$$

where $\quad \mathrm{N}=$ the size of moving window

$\mathrm{j}_{0}=$ initial time of moving window, equal to $\mathrm{k}-\mathrm{N}+1$

By comparing the real and theoretical innovation covariance matrix (Hajiyev et al., 2016; Fu-Mei et al., 2010), IASE modifies the Kalman gain, under the assumption of IASE, in the Kalman update stage, once the measurement model dissatisfies the assumption. As a result, the Kalman gain becomes as Eq.21. Moreover, the measurement error scale factor can be determined by Eq.22.

$$
\begin{gathered}
K=P^{-} H^{T}\left(H P^{-} H^{T}+S R\right)^{-1} \\
S=\left(\hat{C}_{v^{-}}-H P^{-} H^{T}\right) R^{-1}
\end{gathered}
$$

The scale factor of measurement error is equal to an identity matrix in a common Kalman Filter, while in an Adaptive Kalman Filter it becomes a diagonal matrix responsible for enlarging the elements in $\mathrm{R}$ matrix. In other words, $\mathrm{S}$ balances the system by giving another scale to adjust the elements in R matrix, weighting, depending on the configuration of measurement, the importance of each elements in covariance matrix of measurement error. As a result, elements in $\mathrm{S}$ matrix must be larger than one, because it 
is not able to improve the accuracy of measurement, just reduce the influence of distorted ones. Unfortunately, S sometimes becomes a non-diagonal matrix or elements are smaller than one, or even are negative, which violates the assumption and makes the adaption meaningless (Dimirovski et al., 2016). In avoidance of above unwanted condition, another modification must be applied to the scale factor matrix as shown in Eq.23.

$$
\begin{gathered}
S^{*}=\operatorname{diag}\left(s_{1}^{*}, s_{2}^{*}, \ldots s_{n}^{*}\right), \text { where } s_{i}^{*}=\max \left\{1, S_{i i}\right\}, \\
i=1,2, \ldots n
\end{gathered}
$$

On the other hand, in the system stage of Kalman filter, adaption for Q matrix is as well indispensable. A fading matrix is exploited for the adaption of Q matrix (Hajiyev et al., 2016). The concept of $\mathrm{Q}$ matrix adaption is like that for $\mathrm{R}$, they scale the elements of corresponding matrix. In the case of $\mathrm{Q}$ matrix adaption, the covariance matrix of innovation $\hat{C}_{v^{-}}$and the fading matrix $\Lambda$ are derived as follows.

$$
\begin{gathered}
\hat{C}_{v_{k}^{-}}=H_{k}\left(\Phi_{\mathrm{k}} P_{k-1}^{+} \Phi_{k}^{T}+\Lambda_{k} G_{k} Q_{k} G_{k}^{T}\right) H_{k}^{T}+R_{k} \\
\Lambda_{k} \\
=\left(\hat{C}_{v_{k}^{-}}-H_{k} \Phi_{k} P_{k-1} \Phi_{\mathrm{k}}^{\mathrm{T}} H_{k}^{T}\right) \times\left(H_{k} G_{k} Q_{k} G_{k}^{T} H_{k}^{T}\right)^{-1}
\end{gathered}
$$

In the eq.24 and Eq.25, symbols with subscript $\mathrm{k}$ means that information at $\mathrm{k}$ time and $\mathrm{k}-1$ means that at $\mathrm{k}-1$ time. The timestamp is specifically presented here reveals that some information at last time is necessary in this method. Furthermore, in avoidance of incorrect fading matrix generated, the fading matrix is also modified after being generated as shown in Eq.26.

$$
\begin{gathered}
\Lambda^{*}=\operatorname{diag}\left(\lambda_{1}^{*}, \lambda_{2}^{*}, \ldots \lambda_{n}^{*}\right), \text { where } \lambda_{i}^{*}=\max \left\{1, \Lambda_{i i}\right\}, \\
i=1,2, \ldots n
\end{gathered}
$$

where $\quad \Lambda_{i i}=$ Diagonal elements of $\Lambda$ matrix

After the modification of Q matrix, the covariance matrix of state vector in the prediction stage in Kalman Filter changes as:

$$
P_{k}^{-}=\Phi_{k} P_{k-1}^{+} \Phi_{k}^{T}+\Lambda_{k}^{*} G_{k} Q_{k} G_{k}^{T}
$$

The other covariance matching adaptive method based on the residual sequence is Residual-based adaptive scaling estimation (RASE). This approach is almost same as IASE method both compare the difference between actual value and estimated value and modify the covariance matrix of system noise or of measurement error by weighting elements of the matrices, balancing the importance of real configuration. In this scheme, the covariance matrix of residual vector is determined in Eq.28 and the scale matrix is formed as in Eq.29.

$$
\begin{gathered}
\hat{C}_{v^{+}}=\frac{1}{N} \sum_{j=j_{0}}^{k} \mathrm{v}_{\mathrm{j}}^{+} v_{j}^{+T} \\
S=\left(\hat{C}_{v^{+}}+H P^{+} H^{T}\right) R^{-1}
\end{gathered}
$$

The scale matrix of this scheme follows the same rules mentioned in Eq.23. In the procedure of Q adaption of RASE scheme, much inverse matrix calculation is included, causing increase of computation burden and inefficiency of whole system. Consequently, the adaption of covariance matrix of system noise is not presented and discussed in this paper.

\section{EXPERIMENT SETUP}

A field test was carried out in the Taipei City, Taiwan, a large and busy city where hundreds of modern buildings are located for the purpose of evaluating the performance and the efficacy of proposed integration scheme. In the field test, navigation mission had met several tough challenges in this scenario. The testing system contains a low cost Microelectromechanical System (MEMS) IMU and a single frequency GNSS receiver. For INS part, a STIM 300 from Sensonor, which is featured with threeaxis gyroscope and accelerometer was used, and its specifications are listed in Table 1. For GNSS antenna, GPS-702-GG from NovAtel is equipped on the top of land vehicle. In addition, a barometer and an odometer were used as aiding sensors.

About the ground truth system for performance validation was equipped with a higher grade IMU to generate reliable reference solution. For high tactical-grade IMU, NovAtel SPAN@ LCI is applied and its specifications is also listed in Table 1. The specifications of GNSS is same for both system and the detail are skipped because it is not the major work in this paper. Both IMUs were placed on the top of the land vehicle in order to be as closer to GNSS receiver as possible, eliminating the lever arm effect which would cause system error between IMU and the receiver. Figure 7 shows the configuration of experiment platform on the land vehicle.

\begin{tabular}{ccc}
\hline Item & $\begin{array}{c}\text { LCI } \\
\text { Reference }\end{array}$ & $\begin{array}{c}\text { STIM300 } \\
\text { Target }\end{array}$ \\
& & \\
\hline \multicolumn{3}{c}{ Gyroscope } \\
\hline Data rate & $250 \mathrm{~Hz}$ & $250 \mathrm{~Hz}$ \\
Scale Factor & 100 & 500 \\
Bias Instability & 0.05 & 0.15 \\
Angular Random & 0.012 \\
Walk & Accelerometer \\
\hline Date rate & 250 & 250 \\
\hline Scale Factor & 100 & 300 \\
Bias Instability & 0.1 & 0.05 \\
Velocity Random & 0.06 & 0.07 \\
Walk & & \\
\hline Table 1. Specification of LCI and STIM300
\end{tabular}

Table 1. Specification of LCI and STIM300

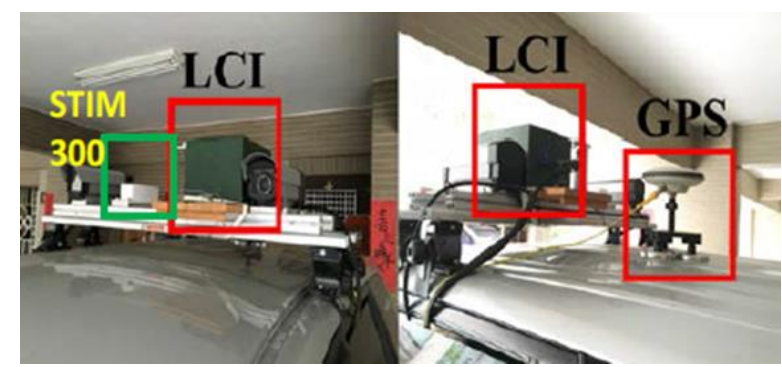

Figure 7. Experiment platform on vehicle

The fieldwork was in downtown of Taipei City and the satellite image of experiment area captured on Google Earth pro is shown in Figure 8. The experiment route started in open area for initial alignment and then entered the urban area. This area was full of harsh and GNSS-unfriendly environments such as overpasses, urban canyon, thread-of-sky and half-of-sky. The street view of these scenarios is illustrated in Figure 8. Under the challenging condition, the land navigator suffered from the poor observability of satellite signals, because they were sheltered, reflected and distorted by the surrounding buildings. 

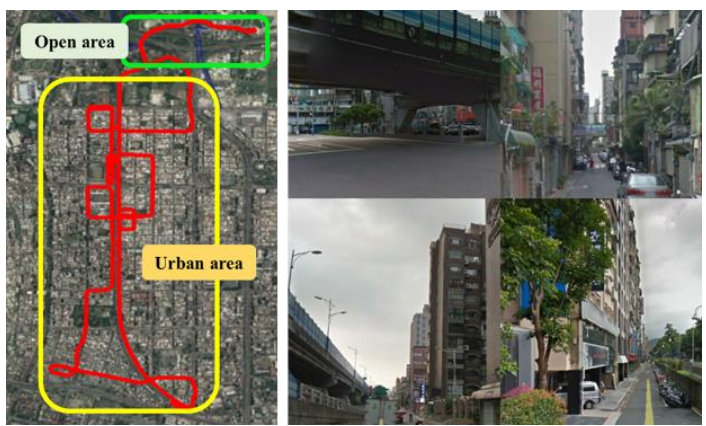

Figure 8. Experiment trajectory (left) and street views (right)

GNSS result also depends on the number of visible satellite, as just mentioned, the amount of available satellite decreases significantly, resulting worse positioning result and even no positioning solution if the number of available satellite is less than 4 .

\section{RESULT AND ANALYSIS}

The result evaluation compares the performance of 4 integration schemes, pure TC scheme, proposed scheme based on innovation (IBASE), proposed scheme based on residuals (RBASE) and ground truth system to be a reference solution. IBASE and RBASE are proposed to verify the performance of different mechanism in adaptive strategies. Besides, these two schemes will be compared with pure TC integration structure to assess the promotion and enhancement issued by the new algorithm in Kalman Filter. The navigation trajectories are compared together on Google Earth Pro. As shown in Figure 9 and Figure 10, 2 practical cases in urban area are presented. With the adaptive strategies and aiding sensors, navigator is able to prevent the positioning result from contamination of multipath effect. In the Figure 9 and Figure 10, there are obvious abnormal jumps in trajectory segment while the vehicle passed though high buildings.

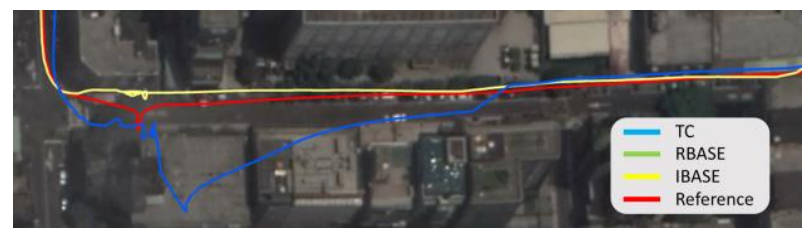

Figure 9. Urban canyon case - I

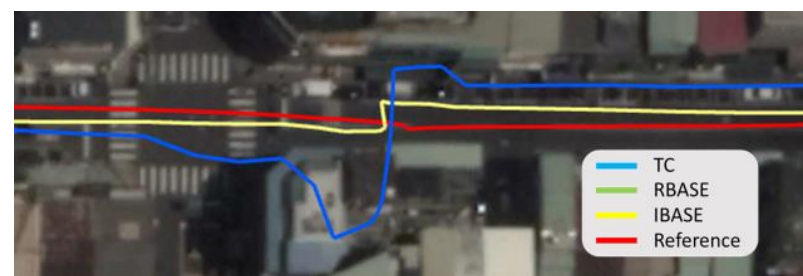

Figure. 10 Urban canyon case - II

One of the most important elements is height information, which is required to be accurate in many future ITS applications, especially for precise $3 \mathrm{D}$ positioning and location finding in urban area. Figure 11 shows the series of solution in vertical for 4 solution. Obviously, the oscillation of pure TC is significant due to the bad signal observability of urban canyons. Some large offsets appear frequently during field test and most of them are larger than 50m; as for the two proposed scheme, it seems and adaptive and aiding sensors help to reduce the maximum offset and make it more stable. Both proposed algorithms improve the height estimation result, though there are offsets remained.

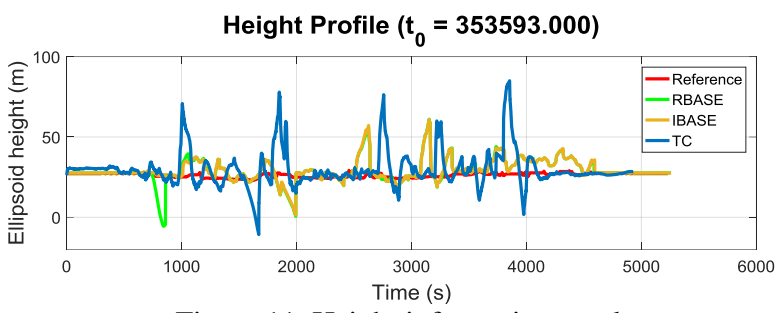

Figure 11. Height information result

It can be seen that in Figure 11, although both RBASE and IBASE schemes is able to suppress the oscillation of height component during the whole trip, there are some peaks that can not be eliminated. The possible reason is the parameters setting for the adaptive scheme and the bad measurement detection. Compare with the high-end reference system, the proposed method need more improvement and test. Besides, series of estimation result, the statistical index was applied to evaluate the statistical characteristics as well. Table 2 shows the maximum error during experiment while Table 3 presents the RMSE result compared to reference solution. In Table 2, after adoption of proposed navigation algorithm on pure $\mathrm{TC}$, the maximum error is reduced by a large level. The proposed scheme prevents the navigation resolution from heavy deterioration. The sensor fusion structure efficiently lower the opportunity of GNSS outage and keep frequently calibrating the IMU error, which also avoids the maximum error growing up within short time.

\begin{tabular}{|ccc|c|}
\hline Max Error $(\mathbf{m})$ & TC & RBASE & IBASE \\
\hline East & 481.085 & 28.462 & 39.750 \\
North & 88.760 & 11.679 & 19.850 \\
Horizontal & 489.205 & 30.765 & 44.431 \\
Up & 56.584 & 7.096 & 35.775 \\
\hline \hline 3D & $\mathbf{4 8 9 . 7 9 4}$ & $\mathbf{2 9 . 7 5 9}$ & $\mathbf{5 0 . 3 2 1}$ \\
\hline
\end{tabular}

Table 2. Max error of TC RBASE and IBASE scheme

\begin{tabular}{|ccc|c|}
\hline RMSE $(\mathbf{m})$ & TC & RBASE & IBASE \\
\hline East & 29.101 & 4.871 & 3.516 \\
North & 5.857 & 3.385 & 2.238 \\
Horizontal & 29.685 & 5.932 & 4.168 \\
Up & 11.906 & 2.489 & 7.247 \\
\hline \hline 3D & 31.984 & $\begin{array}{l}6.433 \\
(80 \%)\end{array}$ & $\begin{array}{l}8.360 \\
(74 \%)\end{array}$ \\
\hline
\end{tabular}

Table 3. RMSE of TC RBASE and IBASE scheme

In Table 3, RMSE is another important index for the evaluation of urban area positioning, navigation solution. The smaller RMSE value means more stable of navigation. The RMSE were calculated compared with the reference result. In Table 3, pure TC gets large RMSE value both in horizontal and vertical directions, and then resulted in 30m RMSE in 3D; RBASE and IBASE lead to tremendous improvement in overall direction. RBASE shows $6 \mathrm{~m}$ in horizontal, $2.5 \mathrm{~m}$ in vertical and $6 \mathrm{~m}$ in $3 \mathrm{D}$ positioning; IBASE presents $4 \mathrm{~m}$ in horizontal, $7 \mathrm{~m}$ in vertical and $8 \mathrm{~m}$ in $3 \mathrm{D}$. In addition, RBASE reaches $80 \%$ improvement from pure TC and IBASE reaches $74 \%$ in $3 \mathrm{D}$ positioning result. 


\section{CONCLUSION}

To enhance the performance of INS/GNSS integration system with external aiding sensors and adaptive strategies is developed in this paper. In the field test, about $80 \%$ improvement in positioning solution is reached. Besides, maximum errors are significantly limited, indicating proposed AKF does INS error calibration more frequently than traditional ones. Positioning accuracy can reach around $6 \mathrm{~m}$ in horizontal direction, $2.5 \mathrm{~m}$ in the vertical and $6 \mathrm{~m}$ in $3 \mathrm{D}$ assessments.

Since the quality of measurement differs quickly and degrades significantly due to the fast--changing outer environment, especially in urban area. The proposed INS/GNSS scheme not only replaces the traditional $\mathrm{LC}$ with $\mathrm{TC}$, but also integrates aiding sensors into Kalman Filter. Furthermore, in this paper adaptive method for TC structure is implemented as well. In order to achieve the high demand of accuracy for intelligent city, authors modified system model, measurement model and update loop in KF, trying to make a great balance in Kalman Filter. Despite the 3D accuracy is not good enough for future application, large improvement and aiding sensors make it a best beginning to achieve seamless navigation.

For the future work, the fusion structure of proposed algorithm will be further investigated with a view to achieving a better and stricter integration structure. Vehicular constraints, Zero Velocity Update (ZUPT) and Non Holonomic Constraint (NHC), will be implemented to enhance the reliability and accuracy for seamless land navigation.

\section{ACKNOWLEDGEMENTS}

The author would like to present his appreciation to Guang Je Tsai, Zhong Ling Tseng and You Liang Chen for their help on conducting the experiment as well as data processing, and the technical suggestions and financial support (MOST107-2221-E006 -125 -MY3 ) from his advisor, Dr. Kai-Wei Chiang that greatly improves the quality of this manuscript.

\section{REFERENCES}

Almagbile, A., Wang, J., Ding, W., 2010. Evaluating the performance of adaptive Kalman filter method in GPS/INS integration, Journal of Global Positioning Systems, 2010, Vol.9, No.1, pp. 33-40.

Chen, D., 1994. Development of a fast ambiguity search filtering (FASF) method for GPS carrier phase ambiguity resolution, $\mathrm{PhD}$ thesis, University of Calgary, Canada.

Chiang, K. W., 2004. INS/GPS Integration Using Neural Networks for Land Vehicular Navigation Application, PhD thesis, University of Calgary, Canada.

Chiang, K. W., Huang, Y. W., 2008. An intelligent navigator for seamless INS/GPS integrated land vehicle navigation application Applied Soft Computing, 8(1), 722-733.

Chiang, K.W., Duong, T.T., Liao, J.K, 2013. The performance analysis of a real-time integrated INS/GPS vehicle navigation system with abnormal GPS measurement elimination, Sensors, Vol. 13, No. 8, pp.10599-10622.

Dimirovski, G.M., 2016. Complex Systems: Relationships between Control, Communication and Computer. Springer.
Farrell, J. and Barth, M., 1998. The global positioning system and inertial navigation, McGraw-Hill Professional.

Fu-Mei, W., \&Yuan-xi, 2010. An Extended Adaptive Kalman Filtering in Tight Coupled GPS/INS Integration. Survey Review, Vol.42, Issue 316, pp. 146-154.

Gelb, A., 1974. Applied optimal estimation. The Analytic Sciences Corporation, Inc.

Godha, S., 2006. Performance evaluation of low cost MEMSbased IMU integrated with GPS for land vehicle navigation application. Master's thesis, University of Calgary, Canada.

Hajiyev, C., Soken, H.E..., Vural, S.Y., 2016. State Estimation and Control for Low-cost Unmanned Aerial Vehicles, Springer.

Hsu, L. T., Jan, S. S. Groves, P. D., Kubo, N, 2015. Multipath mitigation and NLOS detection using vector tracking in urban environment. GPS Solutions, 19(2), 249-262.

Liu, Y., Fan, X., Lv, C., Wu, J., Li, L., Ding, D., 2017. An Innovative Information Fusion Method with Adaptive Kalman filter for integrated INS/GPS navigation of autonomous vehicles. Mech. Syst. Signal Process.100, 605-616

Misra, P., Enge, P., 2001. Global Positioning System : Signals, Measurements, and Performance, Second Edition, , GangaJamuna Press.

M. Park and Y. Gao, 2008. Error and Performance Analysis of MEMS-based Inertial Sensors with a Low-cost GPS Receiver, Sensors, vol. 8, no. 4, pp. 2240-2261.

Park, J., Lee, D., park, C., 2015. Implement of vehicle navigation system using GNSS, INS, odometer and barometer. Journal of Positioning, Navigation, and Timing 4, 141-150.

Pemg, K. Y., Lin, C. A., Chiang, K. W., 2013. Performance analysis of an AKF based tightly-coupled INS/GNSS integrated scheme with NHC for land vehicular application, Transaction of the Canadian Society for Mechanical Engineering, 37, 503-513.

Petovello, M. G., 2003, Real-time integration of tactical-grade IMU and GPS for high-accuracy positioning and navigation, $\mathrm{PhD}$ thesis, University of Calgary, Canada.

Rogers, R. M., 2003. Applied mathematics in integrated navigation system, American Institute of Aeronautics and Astronautics, Inc.

Seo, J., Lee, H. K., Lee, J. G., \&Park, C. G., 2006. Lever arm compensation for GPS/INS/odometer integrated system, International Journal of Control, Automation, and Systems, Vol.4, No.2, pp. 247-254.

Shin, E. H., 2005, Estimation technique for low-cost inertial navigation, $\mathrm{PhD}$ thesis, University of Calgary, Canada.

Sokolovic, V. S., Dikic, G., Stancic, R., 2013. Integration of INS, GPS, magnetometer and barometer for improving accuracy navigation of the vehicle. Defense Science Journal 63, 451-455.

Sun, R., Yang, Y., Chiang, K. W., Duong, T. T., \& Tsai, G. J., 2016. IMU/GPS/VO Integration for vehicle navigation with height enhancement in urban areas, ISPRS Int. J. Geo-Inf. 2016, 5, x; doi:10.3390/ 
Titterton, D., Weston, J.L., 2004. Strapdown Inertial Navigation Technology, Institution of Engineering and Technology.

Wu, F.M.; Yang, Y.X., 2013. An extended adaptive Kalman filtering in tight coupled GPS/INS integration. Surv. Rev. 42, $146-154$.

Yang. Y., 2008. Tightly coupled MEMS INS/GPS integration with INS aided receiver tracking loops. Ph.D. thesis, University of Calgary, Canada. 